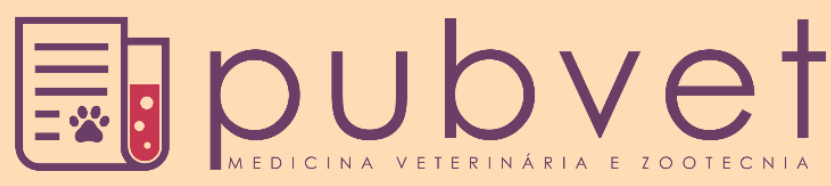

https://doi.org/10.31533/pubvet.v12n12a229.1-7

\title{
Herdabilidade de parâmetros biométricos de Melipona scutellaris Latreille, 1811 (Hymenoptera: Apidae)
}

\author{
Rogério Marcos de Oliveira Alves ${ }^{1 *} \oslash$, Daiane Rodrigues dos Santos ${ }^{2}$, Andreia Santos do \\ Nascimento $^{2}$, Geni da Silva Sodré ${ }^{2} \bullet$, Carlos Alfredo Lopes de Carvalho ${ }^{2} \bullet$ \\ ${ }^{1}$ Professor do Instituto Federal de Educação, Ciência e Tecnologia Baiano, Catu-BA, Brasil. \\ ${ }^{2}$ Universidade Federal do Recôncavo da Bahia, Cruz das Almas-BA, Brasil. E-mail: insecta@ufrb.edu.br \\ *Autor para correspondência, E-mail: eiratama@gmail.com
}

\begin{abstract}
RESUMO. Este trabalho teve por objetivo estimar a herdabilidade no sentido amplo entre os parâmetros biométricos e produtivos: altura dos potes de mel (APM), largura do pote de mel (LPM), volume do pote de mel (VPM), largura dos potes de pólen (LPO), altura dos potes de pólen (APO), peso do pote de pólen (PPP), tamanho da glossa (GLO), comprimento dos favos (CFV) e largura dos favos (LFV), em colônias de Melipona scutellaris. Um total de cento e sessenta e cinco colônias pertencentes a diferentes gerações foram avaliadas. Os resultados foram submetidos à análise de variância, sendo o efeito da interação colônias $\mathrm{x}$ gerações consideradas significativas para a maioria dos parâmetros estudados, a exceção dos parâmetros CFV e LFV. As estimativas de herdabilidade foram: GLO $(0,71)$, LPM $(0,07)$, APM $(0,35)$, VPM $(0,39)$, LPO $(0,14)$, APO $(0,18)$, PPO $(0,27)$, CFV $(0,37)$ e LFV $(0,47)$. Os resultados obtidos possibilitarão ampliação dos estudos e melhoria dos cálculos estatísticos para inclusão em programas de melhoramento da espécie.
\end{abstract}

Palavras chaves: abelhas sem ferrão, meliponicultura, melhoramento genético

\section{Heritability of biometric parameters of Melipona scutellaris Latreille, 1811 (Hymenoptera: Apidae)}

\begin{abstract}
The study aimed to estimate broad sense heritability between biometric parameters and production height of the honey pots (APM), width of the honey pot (LPM), the honey pot volume (VPM), width of the pots of pollen (LPO), height of the pollen pots (APO), weight of the pot of pollen (PPP), size of the glossa (GLO), length of the combs (CFV) and width of the combs (LFV) in colonies of Melipona scutellaris. A total of one hundred sixty-five colonies belonging to different generations were evaluated. The results were submitted to analysis of variance, being the effect of interaction colonies $\mathrm{x}$ generations considered significant for most parameters studied, except the parameters CFV and LFV. The heritability estimates were: GLO (0.71), DPM (0.07), APM (0.35), VPM (0.39), DPO (0.14), APO (0.18), PPP (0.27), CFV (0.37) and LFV (0.47). The resulted obtained allows the expansion of studies and improvement of statistical calculations for inclusion in breeding programs of the species.
\end{abstract}

Keywords: stingless bee, meliponiculture, genetic improvement

\section{Heredabilidad de parámetros biométricos de Melipona scutellaris Latreille, 1811 (Hymenoptera: Apidae)}

RESUMEN. Este trabajo tuvo el objetivo de evaluar el índice de herencia, en sentido amplio, entre los parámetros biométricos y productivos: altura de los potes de miel 
(APM), anchura del pote de miel (LPM), volumen del pote de miel (VPM), anchura de los potes de polen (LPO), altura de los potes de polen (APO), peso del pote de polen (PPP), tamaño de la glosa (GLO), longitud de los panales (CFV) y anchura de los panales (LFV), en colonias de Melipona scutellaris. Un total de ciento y sesenta y cinco colonias pertenecientes a diferentes generaciones fueron evaluadas. Los resultados fueron sometidos a análisis de variancia, siendo el efecto de la interacción colonias vs. generaciones considerado significativo para la mayoría de los parámetros analizados, excepto para los parámetros CFV y LFV. Se estimó la heredabilidad em: GLO $(0,71)$, LPM $(0,07)$, APM $(0,35), \operatorname{VPM}(0,39)$, LPO $(0,14)$, APO $(0,18)$, PPO $(0,27)$, CFV $(0,37)$ y LFV $(0,47)$. Los resultados alcanzados posibilitaran ampliar estudios y mejorar los cálculos estadísticos para su inclusión en programas de mejoramiento de la especie.

Palabras clave: abejas sin aguijón, meliponicultura, mejoramiento genético

\section{Introdução}

O desejo de conseguir pelo melhoramento genético uma abelha ideal que propicie ao homem produtos em quantidade e com qualidade implica na necessidade de conhecer os fatores que interferem na vida de cada espécie, a sua bioecologia. No entanto, o melhoramento genético de abelhas quando comparado ao aplicado a outras espécies animais apresenta algumas diferenças, sendo as estimativas como herdabilidade, semelhança entre parentes e outras difíceis de serem obtidas. Essas diferenças podem ser influenciadas pela estrutura genética interna das colônias, dificultado o processo de melhoramento (Martinez \& Soares 2012). Dessa forma, o trabalho do melhorista é selecionar os genes favoráveis a partir de populações naturais de abelhas.

Abelhas do gêneros Melipona apresentam características no mecanismo de acasalamento que as diferenciam das abelhas Apis mellifera (Aidar 2010; Imperatriz-Fonseca \& Nunes-Silva 2010; Page Júnior et al. 2012). Os estudos dos mecanismos de acasalamento determinaram que as rainhas se acasalem com apenas um zangão na maioria das espécies de meliponíneos, sendo que em M. scutellaris até $8 \%$ das rainhas podem acasalar com 2 zangões durante o vôo nupcial (Carvalho 2001). Este fato sugere que as características transmitidas de geração em geração podem ser mais facilmente monitoradas.

Lush (1940) definiu herdabilidade como sendo a fração da variância observada a qual foi causada pela diferença gênica ou genotípica dos indivíduos, e esta dá uma ideia de quanto uma característica pode ser herdável e quanto ela é influenciada pelo meio (Monteiro \& Lobo 1993). As estimativas de herdabilidade geralmente determinam se os caracteres analisados podem ou não ser aprimorados facilmente por meio do melhoramento genético (Fox et al. 2008). Na busca de informações que subsidiem programas de melhoramento genético de abelhas, geralmente, os estudos apresentam a estimativa de herdabilidade (Rinderer et al. 1983; Collins et al. 1984; Bienefeld \& Pirchner 1990; Costa-Maia et al. 2011; Padilha et al. 2013; Oliveira et al. 2015; Brascamp et al. 2016).

Muitos pesquisadores elaboraram métodos para estimativa de herdabilidade em abelhas (Rinderer et al. 1983; Collins et al. 1984; Bienefeld \& Pirchner 1990). Como exemplo, tem-se o método proposto por Rinderer (1977) com inseminação artificial em um esquema onde se utiliza o sêmen de um macho para inseminar uma fêmea e formar famílias de meias irmãs. No entanto, Oldroyd \& Moran (1983) consideram que este método teria desvantagem na inseminação artificial por ser um processo caro e que consumiria tempo. Eles propuseram um método simples que dispensaria o controle do acasalamento e criação de rainhas. Nesse método o acasalamento seria natural e a determinação da herdabilidade restrita seria evidenciada pela proporção entre variância e coancestria.

Como os valores genéticos determinam o que ocorrerá na próxima geração, mas apenas os valores fenotípicos são os que podem ser mensurados, torna-se necessário conhecer a relação entre ambos como forma de se predizer a viabilidade de um trabalho de melhoramento e quais as melhores características para seleção nas abelhas sem ferrão. Em meliponíneos os estudos de herdabilidade são escassos e concentrados na produção de mel/colônia e utilizando parâmetros morfométricos (Barros 2006; Alves et al. 2012; Oliveira et al. 2015). Brito et al. (2013) para $M$. quadrifasciata anthidioides realizaram mensuração dos parâmetros biométricos da colônia (discos de cria, potes de mel e potes de 
pólen) e de rainha, além de morfometria da glossa de operárias obtendo que o peso da rainha fisogástrica e o comprimento da glossa não apresentaram diferenças entre as gerações e que houve diferença para o número de discos de cria e entre características de produção de mel e pólen, com variabilidade entre as gerações. Entretanto quais as características que deverão ser utilizadas pelo pesquisador no trabalho de campo para obter colônias produtivas?

A partir de parâmetros avaliados por outros pesquisadores (Farrar 1937; Woyke 1984; Souza et al. 2002; Barros 2006; Brito et al. 2013; Oliveira et al. 2015) este trabalho teve por objetivo estimar a herdabilidade entre parâmetros biométricos e produtivos em colônias da abelha Melipona scutellaris.

\section{Material e métodos}

O estudo foi desenvolvido no meliponário do Núcleo de Estudo dos Insetos/Grupo de Pesquisas Insecta, na Universidade Federal do Recôncavo da Bahia (UFRB). A área caracterizase por intensa atividade antrópica, com pomares, plantios de diversas culturas e pastagem. O experimento foi desenvolvido em delineamento inteiramente casualizado com cinco repetições.

Um total de 165 colônias de $M$. scutellaris pertencentes aos Parentais e as gerações F1 e F2 foram avaliados quanto aos parâmetros: altura dos potes de mel (APM) em $\mathrm{cm}$, largura do pote de mel (LPM) cm, volume do pote de mel (VPM) $\mathrm{mL}$, largura dos potes de pólen $(\mathrm{LPO}) \mathrm{cm}$, altura dos potes de pólen $(\mathrm{APO}) \mathrm{cm}$, peso do pote de pólen (PPP) g, tamanho da glossa (GLO) mm, comprimento dos favos (CFV) $\mathrm{cm}$ e largura dos favos (LFV) $\mathrm{cm}$. Os parâmetros foram escolhidos através da pesquisa em trabalhos realizados com abelhas por pesquisadores anteriormente citados e que podem ser utilizados por criadores a campo.

As características externas de diâmetro e altura dos potes de mel $(n=15 /$ colônia $)$ e de pólen ( $\mathrm{n}=15 /$ colônia) foram medidas utilizando uma régua graduada. Para avaliação do peso dos potes de pólen e de mel foi utilizada uma balança digital de precisão. $\mathrm{O}$ volume dos potes de mel foi obtido por meio da sucção do conteúdo dos potes, com auxílio de seringas descartáveis graduadas de $20 \mathrm{~mL}$. Esses parâmetros são os mais utilizados pelos criadores quando da seleção de colônias para produção. Para avaliação do comprimento da glossa foram capturadas e sacrificadas operárias jovens ( $\mathrm{n}=15 /$ colônia $)$ em recipiente contendo acetato de etila, para facilitar a exposição da glossa.

A remoção foi feita com auxílio de pinças e estiletes conforme a metodologia de Mendes et al. (2002). Após a remoção, esta foi colocada em recipiente contendo álcool $46^{\circ} \mathrm{GL}$ para conservação do material até a sua medição. No momento da medição, as estruturas foram colocadas em placa de Petri. Em seguida, adicionou-se água destilada para reidratar o material. A medida foi tomada utilizando lâminas e lupa Olympus modelo SMZ 168, séries com aumento de 0,5 e do programa Motic imagens Plus 2.0 de acordo com as classificações de Viana \& Kleinert (2005). Para o processo de medição foi considerado a medida da ponta da glossa até a base da paraglossa.

Os resultados obtidos foram submetidos à análise de variância considerando como fontes de variação colônias, gerações e o resíduo. Para o estudo dos componentes de variância foi adotado o modelo estatístico:

1. Fatores de variação colônias e gerações

Yijk $=\mathrm{m}+\mathrm{Ci}+\mathrm{Gj}+\mathrm{CGij}+\mathrm{Eijk}$, onde:

Yijk = influência sofrida pela repetição $\mathrm{k}$, devido ao efeito da geração j sobre a colônia i.

$\mathrm{m}$ - média geral

$\mathrm{Ci}$ - efeito da colônia i.

$\mathrm{Gj}$ - efeito da geração $\mathrm{j}$.

CGij - efeito da interação colônias x geração

Eij - erro aleatório associado a cada observação

2. Fator de variação geração

Yjk $=\mathrm{m}+\mathrm{Gj}+$ Eijk, onde:

Yijk = influência sofrida pela repetição $\mathrm{k}$, devido ao efeito da geração j.

$\mathrm{m}$ - média geral

$\mathrm{Gj}$ - efeito da geração j.

Eij - erro aleatório

Posteriormente calculou-se a herdabilidade no sentido amplo $\left(\mathrm{H}^{2}=\right.$ Valor Genotípico/Valor Fenotípico) por meio do programa Genes ( $\underline{\mathrm{Cruz}}$ 2005; Oliveira et al. 2015).

\section{Resultados e discussão}

Os valores da análise de variância e o resultado do teste $\mathrm{F}$ para os parâmetros avaliados considerando os efeitos da colônia e gerações estão descritos na Tabela 1. 
Tabela 1. Análise de variância e valores de significância dos Quadrados Médios (efeito colônia x gerações) dos parâmetros: altura dos potes de mel (APM), largura do pote de mel (LPM) volume do pote de mel (VPM), largura dos potes de pólen (LPO), altura dos potes de pólen (APO), peso do pote de pólen (PPP), tamanho da glossa (GLO), comprimento dos favos (CFV) e largura dos favos (LFV), em colônias de Melipona scutellaris

\begin{tabular}{|c|c|c|c|c|c|c|c|c|c|c|}
\hline \multirow[b]{2}{*}{$\mathrm{FV}$} & \multirow{2}{*}{ GL } & \multicolumn{9}{|c|}{ Quadrados Médios } \\
\hline & & $\begin{array}{l}\text { LPM } \\
(\mathrm{cm})\end{array}$ & $\begin{array}{l}\text { APM } \\
(\mathrm{cm})\end{array}$ & $\begin{array}{l}\text { VPM } \\
(\mathrm{mL})\end{array}$ & $\begin{array}{l}\text { LPO } \\
(\mathrm{cm})\end{array}$ & $\begin{array}{l}\text { APO } \\
(\mathrm{cm})\end{array}$ & $\begin{array}{c}\text { PPP } \\
(\mathrm{g})\end{array}$ & $\begin{array}{l}\text { GLO } \\
(\mathrm{mm})\end{array}$ & $\begin{array}{l}\text { CFV } \\
(\mathrm{cm})\end{array}$ & $\begin{array}{l}\text { LFV } \\
(\mathrm{cm})\end{array}$ \\
\hline Colônias & 50 & $0,87 \mathrm{~ns}$ & $1,82 *$ & $57,04 *$ & $2,94 \mathrm{~ns}$ & $6,01 \mathrm{~ns}$ & $135,66 \mathrm{~ns}$ & $0,15 * *$ & $25,45^{*}$ & $22,56 * *$ \\
\hline Geração & 2 & $1,48 \mathrm{~ns}$ & $5,15 *$ & $123,25 *$ & $15,51 * *$ & $10,48 \mathrm{~ns}$ & $599,79 * *$ & $7,01 * *$ & $63,32 *$ & $137,78 * *$ \\
\hline Colônias x Geração & 100 & $0,81^{* *}$ & $1,17 * *$ & $34,77 * *$ & $2,52 * *$ & $4,84 * *$ & $97,90 * *$ & $0,43 * *$ & $15,9 \mathrm{~ns}$ & $11,90 \mathrm{~ns}$ \\
\hline Erro & 612 & 0,17 & 0,29 & 9,23 & 0,45 & 0,745 & 22,76 & 0,15 & 12,60 & 11,08 \\
\hline Média & - & 2,81 & 3,57 & 15,58 & 2,46 & 3,11 & 13,96 & 2,62 & 9,33 & 8,63 \\
\hline $\mathrm{CV}$ & - & 14,75 & 15,16 & 19,5 & 27,18 & 27,72 & 34,15 & 3,43 & 38,05 & 38,13 \\
\hline
\end{tabular}

ns: não significativo. $* \mathrm{e}^{* *}$ significativo pelo teste $\mathrm{t}$, a $5 \%$ e $1 \%$ de probabilidade respectivamente.

$\mathrm{O}$ teste $\mathrm{F}$ não foi significativo para a largura dos potes de mel (LPM), altura dos potes de pólen (APO) e peso dos potes de pólen (PPP) quando os fatores de variação foram colônias e geração (Tabela 1).

$\mathrm{O}$ efeito da interação colônias $\mathrm{x}$ gerações foi considerado significativo para a maioria dos parâmetros estudados, a exceção dos parâmetros CFV e LFV que não apresentaram diferença entre as médias, demonstrando que existe potencial para seleção de colônias a partir dos parâmetros estudados.

A análise dos efeitos de colônia e geração pode ser visualizada na Tabela 1. A análise das gerações, como fonte de variação, demonstra significância para todos os parâmetros, com aumento do erro e do CV (Tabela 2). Os valores do CV para o parâmetro tamanho glossa (GLO) foram considerados baixos $(4,64)$. Entretanto, para os demais caracteres situou-se entre 10 a $40 \%$. Coeficiente de variação inferior a $10 \%$ demonstram uma boa precisão experimental (Gomes 1985). De acordo com Fonseca \& Kerr (2006) coeficientes de variação elevados em insetos são comuns, pois as medidas apresentam uma grande variação. Medidas de parâmetros bionômicos baseados na arquitetura dos ninhos em abelhas sem ferrão propiciam estimativas pouco precisas, resultando em CV alto devido à variação apresentada.
Uma informação relevante para o melhorista é conhecer a variação fenotípica atribuída a causas genéticas e ambientais. Sendo a fonte de variação geração apenas, observa-se um menor número de graus de liberdade indicando menor precisão e maior efeito do ambiente. Quando o ambiente interfere em grande magnitude a eficácia da predição do valor genotípico por meio do valor fenotípico é reduzida. Quanto da natureza genética, técnicas mais simples de melhoramento, como a seleção massal, podem ser aplicadas, proporcionando ganhos consideráveis pela seleção (Cruz 2005).

$\mathrm{Na}$ interação colônia x geração os valores dos efeitos sobre os parâmetros são menores devido ao número de GL, demonstrando uma distribuição da influência ambiental sobre os parâmetros. Quando são analisados a ANOVA tendo as gerações como tratamento os valores de GL aumentam o valor do erro devido a menor precisão das medidas e a forte influência ambiental. $\mathrm{O}$ uso das gerações como única fonte de variação proporcionará maior efeito ambiental e reduzirá a precisão do experimento. Fica evidente que nesse caso a influência ambiental pode dificultar o reconhecimento dos genótipos superiores, igualando dispares e discriminando idênticos (ㄷuz 2005).

Tabela 2. Análise de variância e valores de significância dos Quadrados Médios (efeito gerações) dos parâmetros: altura dos potes de mel (APM), largura do pote de mel (LPM), volume do pote de mel (VPM), largura dos potes de pólen (LPO), altura dos potes de pólen (APO), peso do pote de pólen (PPP), tamanho da glossa (GLO), comprimento dos favos (CFV) e largura dos favos (LFV) em colônias de Melipona scutellaris

\begin{tabular}{lcccccccccc}
\hline & & \multicolumn{10}{c}{ Quadrados Médios $(\mathrm{G})$} \\
\cline { 3 - 10 } & FV & GLM & APM & VPM & LPO & APO & PPP & GLO & CFV & $\begin{array}{c}\text { LFV } \\
(\mathrm{cm})\end{array}$ \\
& & $(\mathrm{cm})$ & $(\mathrm{cm})$ & $(\mathrm{mL})$ & $(\mathrm{cm})$ & $(\mathrm{cm})$ & $(\mathrm{g})$ & $(\mathrm{mm})$ & $(\mathrm{cm})$ & $(\mathrm{cm})$ \\
\hline Geração & 2 & $1,48^{* *}$ & $5,15^{* *}$ & $123,25^{* *}$ & $15,51^{* *}$ & $10,48^{* *}$ & $599,70^{* *}$ & 7,01 & $63,32^{*}$ & $137,78^{* *}$ \\
Erro & 762 & 0,30 & 0,51 & 15,72 & 0,88 & 1,63 & 40,03 & 0,28 & 13,87 & 11,94 \\
CV & - & 19,58 & 19,96 & 25,44 & 38,13 & 41,05 & 45,29 & 4,64 & 39,93 & 40,04 \\
\hline
\end{tabular}

ns : não significativo.* e $* *$ significativo pelo teste $\mathrm{t}$, a $5 \%$ e $1 \%$ de probabilidade respectivamente. 
As estimativas das herdabilidades variaram de acordo com os parâmetros mensurados (Tabela 3), sendo que na avaliação da herdabilidade ampla, através da análise estatística realizada nesse trabalho, a maior influência foi dos fatores ambientais. O LPM apresentou baixo valor de herdabilidade $(0,07)$ entre geração e colônias demonstrando ser uma característica que sofre forte influência ambiental. Devido ao seu processo de formação que é resultante do espaço existente no sítio de nidificação, sendo isso visualizado em colônias alojadas em abrigos naturais onde o espaço é restrito e desuniforme. Potes construídos em melgueiras racionais apresentam menor variação no tamanho e forma. Apesar de apresentar 0,35 de valor de herdabilidade, APM reflete o que foi descrito para o LPM. Esse valor é decorrente da maior precisão de medida dos parâmetros, o que acarretou um menor valor do erro experimental.

Tabela 3. Estimativas das herdabilidades quando analisados os efeitos colônias x geração e efeito geração sobre os parâmetros: largura dos potes de mel $(\mathrm{LPM}) \mathrm{cm}$, altura do pote de mel (APM) cm, volume do pote de mel (VPM) $\mathrm{mL}$, largura dos potes de pólen (LPO) $\mathrm{cm}$, altura dos potes de pólen (APO) cm, Peso dos potes de pólen (PPP) g, tamanho da glossa (GLO) mm, comprimento dos favos (CFV) $\mathrm{cm}$ e largura dos favos (LFV) $\mathrm{cm}$

\begin{tabular}{lcc}
\hline \multirow{2}{*}{ Parâmetros Herdabilidade } \\
\cline { 2 - 3 } & Colônias x Geração & Geração \\
\hline LPM & 0,07 & 0,79 \\
APM & 0,35 & 0,90 \\
VPM & 0,39 & 0,87 \\
LPO & 0,14 & 0,94 \\
APO & 0,18 & 0,84 \\
PPP & 0,27 & 0,93 \\
GLO & 0,71 & 0,99 \\
CFV & 0,37 & 0,78 \\
LFV & 0,47 & 0,91 \\
\hline
\end{tabular}

A análise para VPM indica 0,39 do valor da herdabilidade. Apesar do maior valor em relação à APM e LPM este fator está estreitamente relacionado a ambos. É um critério de relevante importância quando estamos selecionando colônias para a produção de mel, uma vez que potes de maior volume facilitam a colheita do produto e propicia economia de energia na colônia.

A estimativa de herdabilidade para a largura dos potes de pólen (LPO) e altura dos potes de pólen (APO) apresentaram respectivamente 0,14 e 0,18 de estimativa de herdabilidade (Tabela 3 ).
Dessa forma essas características, como no caso de LPM e APM, podem ser consideradas de baixa herdabilidade por estarem sujeitas, na sua maior parte, à forte influência dos fatores ambientais.

O peso dos potes de pólen (PPP) é uma característica que apresenta influência do LPO e APO. Quanto maior os valores dessas características maiores será a média do PPP, mesmo mantendo constante o número de potes de armazenamento de pólen. Apresentando 0,27 de estimativa de herdabilidade essa característica possui valor considerado médio na escala de herdabilidade.

Esse parâmetro (PPP) é muito importante, pois afeta diretamente a produção de pólen, podendo ser ampliada a herdabilidade através da seleção de rainhas em colônias com potes de pólen mais pesados. Fonseca \& Kerr (2006) calcularam a herdabilidade para produção de pólen em 0,72 de variações genotípicas para $A$. mellifera.

O CFV e LFV apresentaram herdabilidade de 0,37 e 0,47 entre colônias e gerações sendo considerado um exemplo da combinação de fatores genéticos com alta contribuição do ambiente. Alves et al. (2007) e Souza et al. (2008) demonstraram que o tamanho de favos entre dois meliponíneos estudados apresentaram regularidade nas medidas mesmo após divisão e que estas são dependentes do espaço ocupado e manejo da colônia nas diferentes épocas do ano.

A estimativa de herdabilidade entre colônias demonstrou que a GLO possui o mais alto coeficiente de herdabilidade $(0,71)$ entre as características apresentadas nesse trabalho quando avaliado o efeito das colônias, sendo altamente significativo para todos os efeitos entre colônias e gerações e sua interação. Isso demonstra ser, uma característica de alta herdabilidade e com baixa influência de fatores ambientais na sua formação. Souza et al. (2002) encontraram altas estimativas de herdabilidade para uma seleção entre rainhas mães $(0,52)$, entre rainhas filhas dentro de rainhas mães $(0,62)$ e seleção massal entre rainhas filhas $(0,74)$ para glossa.

Ao analisar a herdabilidade apenas com efeito das gerações a glossa apresentou valor alto de 0,99 (Tabela 3). Esse resultado demonstra que para o efeito geração esse parâmetro tem forte influência genética; além de ser uma 
característica cuja precisão das medidas é alta, possibilitando assim maior precisão do cálculo da herdabilidade devido à homogeneidade dos dados. Bienefeld \& Pirchner (1990) propuseram estimar uma herdabilidade para efeitos de rainha e outra para efeitos de operárias. Estes cálculos podem ser utilizados em meliponíneos, entretanto não seria possível o cálculo da herdabilidade restrita devido à dificuldade de calcular os valores referentes à decomposição da variância genética. Outro problema diz respeito à unidade utilizada para obtenção da estimativa, se a colônia ou o indivíduo. Carvalho et al. (2001) afirmam que a unidade utilizada para obtenção da herdabilidade será aquela sobre a qual a seleção é praticada. Devido a abelhas viverem em comunidades de indivíduos aparentados, Collins et al. (1984) ressaltam que a depender da unidade escolhida para o cálculo da herdabilidade pode haver aumento da covariância entre indivíduos e levar a uma redução na precisão das estimativas.

Valores maiores de herdabilidade foram obtidos quando as medidas são realizadas em características de mensuração mais precisas. Rinderer (1977) cita que as características de interesse econômico são resultantes da ação combinada de muitas operárias, esse fato indica uma predisposição de utilizar a colônia como unidade de trabalho e não o indivíduo. Possuindo assim uma variação individual e em nível de colônia (Loengarov \& Tereshko 2008; Stanimirović et al. 2010). Além disso, mesmo que haja controle dos fatores ambientais sempre restará alguma variação intangível de responsabilidade de fatores do meio ambiente (Gomes 1985). Harbo \& Harris (1999) sugerem que valores de herdabilidade superiores a 0,25 indicam potencial de seleção. Dessa forma, uma vez que a seleção pode afetar a determinação da expressão genética das características, os valores de herdabilidade superiores a 0,25 encontrados nesse trabalho demonstram que os meliponicultores podem utilizar essas características como critério de seleção.

\section{Conclusão}

Apesar da dificuldade no cálculo da herdabilidade devido a influência ambiental e a aplicação do resultado em colônias de $M$. scutellaris fica evidenciado que alguns parâmetros podem ser utilizados como critério de seleção de colônias para produção de mel dessa espécie de meliponíneo.

\section{Agradecimentos}

Este estudo foi financiado em parte pela "Coordenação de Aperfeiçoamento de Pessoal de Nível Superior - Brasil" (CAPES) - Código Financeiro 001 e pelo "Conselho Nacional de Desenvolvimento Científico e Tecnológico" (CNPq) pela concessão de bolsa para CALC (número 305885/2017-0).

\section{Referências bibliográficas}

Aidar D.S. 2010. A mandaçaia: biologia e manejo de abelhas com ênfase à Melipona quadrifasciata Lep (Hymenoptera, Apidae, Meliponinae). FUNPEC-Editora, Ribeirão Preto, São Paulo, Brasil.

Alves R.M.O., Carvalho C.A.L., Faquinello P., Ledo C.A.S. \& Figueredo L. 2012. Parâmetros biométricos e produtivos de colônias de Melipona scutellaris Latreille, 1811 (Hymenoptera: Apidae) em diferentes gerações. Magistra 24, 105-111.

Alves R.M.O., Souza B.A. \& Carvalho C.A.L. 2007. Notas sobre a bionomia de Melipona mandacaia (Apidae: Meliponina). Magistra 19, 204-212.

Barros J.R.S. 2006. Genetic breeding on the bee Melipona scutellaris (Apidae, Meliponinae). Acta Amazonica 36, 115-120.

Bienefeld K. \& Pirchner F. 1990. Heritabilities for several colony traits in the honeybee (Apis mellifera carnica). Apidologie 21, 175-183.

Brascamp E.W., Willam A., Boigenzahn C., Bijma P. \& Veerkamp R.F. 2016. Heritabilities and genetic correlations for honey yield, gentleness, calmness and swarming behaviour in Austrian honey bees. Apidologie 47, 739-748.

Brito B.B.P., Faquinello P., Paula-Leite M.C. \& Carvalho C.A.L. 2013. parâmetros biométricos e produtivos de colônias em gerações de Melipona quadrifasciata anthidioides. Archivos de Zootecnia 62, 265-273.

Carvalho F.I.F., Silva S.A., Kurek A.J. \& Marchioro V.S. 2001. Estimativas e implicações da herdabilidade como estratégia de seleção. UFPEL Gráfica Universitária, Pelotas, Rio Grande do Sul, Brasil.

Carvalho G.A. 2001. The number of sex alleles (CSD) in a bee population and its practical importance (Hymenoptera: Apidae). Journal of Hymenoptera Research 10, 10-15.

Collins A.M., Rinderer T.E., Harbo J.R. \& Brown M.A. 1984. Heritabilities and correlations for several characters in the honey bee. Journal of Heredity 75, 135-140. 
Costa-Maia F.M., Toledo V.A.A., Martins E.N., LinoLourenço D.A., Sereia M.J., Oliveira C.A.L., Faquinello P. \& Halak A.L. 2011. Estimates of covariance components for hygienic behavior in africanized honeybees (Apis mellifera). Revista Brasileira de Zootecnia 40, 1909-1916.

Cruz C.D. 2005. Princípios de genética quantitativa. Universidade Federal de Viçosa, Viçosa, Minas Gerais, Brasil.

Farrar C.L. 1937. The influence of colony populations on honey production. Journal of Agricultural Research 54, 945-954.

Fonseca V.M.O. \& Kerr W.E. 2006. Influência da troca de rainhas entre colônias de abelhas africanizadas na produção de pólen. Bioscience Journal 22, 107-118.

Fox G.P., Bowman J., Kelly A., Inkerman A., Poulsen D. \& Henry R. 2008. Assessing for genetic and environmental effects on ruminant feed quality in barley (Hordeum vulgare). Euphytica 163, 249257.

Gomes F.P. 1985. Curso de estatística experimental. ESALQ/NOBEL, Piracicaba, São Paulo, Brasil.

Harbo J.R. \& Harris J.W. 1999. Heritability in honey bees (Hymenoptera: Apidae) of characteristics associated with resistance to Varroa jacobsoni (Mesostigmata: Varroidae). Journal of Economic Entomology 92, 261-265.

Imperatriz-Fonseca V.L. \& Nunes-Silva P. 2010. As abelhas, os serviços ecossistêmicos e o Código Florestal Brasileiro. Biota Neotropica 10, 59-62.

Loengarov A. \& Tereshko V. 2008. Phase transitions and bistability in honeybee foraging dynamics. Artificial Life 14, 111-120.

Lush J.L. 1940. Intra-sire correlations or regressions of offspring on dam as a method of estimating heritability of characteristics. Proceedings of the American Society of Animal Nutrition 1940, 293301.

Martinez O.A. \& Soares A.E.E. 2012. Melhoramento genético na apicultura comercial para produção da própolis. Revista Brasileira de Saúde e Produção Animal 13, 982-990.

Mendes C.T., Espindola S.M.C.G., Espindola F.S., Lima A.B.P. 2002. Neurobiologia molecular em abelhas Apis mellifera. Horizonte Científico 1, 1-24.

Monteiro S.G. \& Lobo R.B. 1993. Métodos para estimativa de coeficiente de herdabilidade nas abelhas. Zootecnia 31, 113-124.

Oldroyd B. \& Moran C. 1983. Heritability of worker characters in the honeybee (Apis mellifera).
Australian Journal of Biological Sciences 36, 323332.

Oliveira K.N., Paula-Leite M.C., Faquinello P., Carvalho C.A.L., Lino-Lourenço D.A., Sampaio R.B. \& Santos E.B. 2015. Parâmetros genéticos para características produtivas e biométricas em abelha Melipona quadrifasciata anthidioides Lepeletier. Arquivo Brasileiro de Medicina Veterinária e Zootecnia 67, 819-826.

Padilha A.H., Sattler A., Cobuci J.A. \& McManus C.M. 2013. Genetic parameters for five traits in Africanized honeybees using Bayesian inference. Genetics and Molecular Biology 36, 207-213.

Page Júnior R.E., Rueppell O. \& Amdam G.V. 2012. Genetics of reproduction and regulation of honeybee (Apis mellifera L.) social behavior. Annual Review of Genetics 46, 97-119.

Rinderer T.E. 1977. Measuring the heritability of characters of honeybees. Journal of Apicultural Research 16, 95-98.

Rinderer T.E., Collins A.M. \& Brown M.A. 1983. Heritabilities and correlations of the honey bee: response to Nosema apis, longevity, and alarm response to isopentyl acetate. Apidologie 14, 79-85.

Souza B.A., Carvalho C.A.L. \& Alves R.M.O. 2008. Notas sobre a bionomia de Melipona asilvai (Apidae: Meliponini) como subsídio à sua criação racional. Archivos de Zootecnia 57, 53-62.

Souza D.C., Cruz C.D., Campos L.A.O. \& Regazzi A.J. 2002. Correlation between honey production and some morphological traits in africanized honey bees (Apis mellifera). Ciência Rural 32, 869-872.

Stanimirović Z., Stevanović J., Aleksić N. \& Stojić V. 2010. Heritability of grooming behaviour in grey honey bees (Apis mellifera carnica). Acta Veterinaria 60, 313-323.

Viana B.F. \& Kleinert A.d.M.P. 2005. A community of flower-visiting bees (Hymenoptera: Apoidea) in the coastal sand dunes of northeastern Brazil. Biota Neotropica 5, 79-91.

Woyke J. 1984. Correlations and interactions between population, length of worker life and honey production by honeybees in a temperate region. journal of Apicultural Research 23, 148156.

Recebido: 23 outubro, 2018.

Aprovado: 16 novembro, 2018

Publicado: 27 dezembro, 2018.

Licenciamento: Este artigo é publicado na modalidade Acesso Aberto sob a licença Creative Commons Atribuição 4.0 (CC-BY 4.0), a qual permite uso irrestrito, distribuição, reprodução em qualquer meio, desde que o autor e a fonte sejam devidamente creditados. 